\title{
Estimating tree aboveground biomass using multispectral satellite-based data in Mediterranean agroforestry system using random forest algorithm
}

\author{
Patrícia Lourenço $^{\text {a,b,c,*, Sérgio Godinho }}{ }^{\text {d,e }}$, Adélia Sousa ${ }^{\text {a }}$, Ana Cristina Gonçalves ${ }^{\text {a }}$ \\ ${ }^{\text {a }}$ MED - Mediterranean Institute for Agriculture, Environment and Development, Departamento de Engenharia Rural, Escola Ciências e Tecnologia, Universidade de Évora, \\ Pólo da Mitra, Ap. 94, 7006-554, Évora, Portugal \\ b Agroinsider Lda., PITE, R. Circular Norte, NERE, Sala 18, 7005-841, Évora, Portugal \\ ${ }^{\mathrm{c}}$ CICGE - Centro de Investigação Em Ciências Geo-Espaciais, Faculdade de Ciências da Universidade Do Porto, Observatório Astronómico Prof. Manuel de Barros, \\ Alameda Do Monte da Virgem, 4430-146, Vila Nova de Gaia, Portugal \\ ${ }^{\mathrm{d}}$ Institute of Earth Sciences, University of Évora, 7000-671, Évora, Portugal \\ ${ }^{\mathrm{e}}$ EaRSLab-Earth Remote Sensing Laboratory, University of Évora, 7000-671, Évora, Portugal
}

\section{A R T I C L E I N F O}

\section{Keywords:}

Remote sensing

Very high spatial resolution images

Vegetation mask

Vegetation indices

Textural features

\begin{abstract}
A B S T R A C T
Forest aboveground biomass (AGB) is a key biophysical variable to assess and monitor the spatio-temporal changes of forest ecosystems. AGB should be accurately and timely estimated through remote sensing to provide valuable information to better support sustainable forest management strategies. QuickBird and WorldView2 satellites data and Random Forest (RF) regression model were used to estimate tree AGB in Mediterranean agroforestry systems. Spectral bands, vegetation indices and Grey-Level Co-occurrence Matrix (GLCM) texture features of 140 plots with and without vegetation mask were used as independent variables, while total of AGB per plot was used as dependent variable. A model with good performance was obtained for a complex agroforestry system, with an $\mathrm{R}^{2}$ of $82.0 \%$ and RMSE of $10.5 \mathrm{t} /$ ha $(22.6 \%)$. The top 11 most important variables have $80.3 \%$ of total relative importance, with $59.6 \%$ of GLCM textural features, $12.3 \%$ of vegetation indices and $8.4 \%$ of spectral bands. The results highlight the importance of the variable GLCM texture, and the use of vegetation mask and RF regression model to collect accurate spatial information on key crown cover attributes, by excluding the spectral contribution of understory vegetation and soil characteristic, of Mediterranean agroforestry systems.
\end{abstract}

\section{Introduction}

Forest aboveground biomass (AGB) is a key biophysical variable for estimating carbon and water cycling, and energy fluxes between land surface and atmosphere layers which are relevant processes on the background of climate change (Kumar and Mutanga 2017; Urbazaev et al., 2018). Moreover, AGB is a fundamental variable for assessing and monitoring the spatio-temporal changes of structural and functional attributes of forest ecosystems (Alves et al., 2010; ESA 2012; Gonçalves, Sousa, and Gonçalves et al., 2019). Therefore, as an important biophysical variable for both climate change and forest ecosystems quality and productivity assessment, accurately and timely AGB estimations are needed to provide valuable information to better support sustainable forest management strategies (Gallaun et al., 2010; Wu et al., 2016).

The frequently used methods to estimate AGB are the indirect, based on statistical relationships between biomass (dependent variable) and one or a few easy-to-measure tree variables (independent variables), which in turn, are based on field measurements (Correia et al., 2008; Paulo and Tomé, 2006). These methods use forest inventories and allometric functions at tree level to evaluate biomass at the plot level, and an extrapolation method to estimate AGB for the whole study area (Fehrmann and Kleinn 2006; McRoberts et al., 2010). However, while the traditional field-based measurements provide the most accurate AGB estimations, these methods are time-consuming, labor-intensive, and better suited for small areas (Kumar and Mutanga 2017; Lu et al., 2016; Wang et al., 2016). During the last decades, valuable remote sensing (RS) approaches have been developed to estimate AGB and assess its spatio-temporal variability (Eisfelder et al. 2012; Shao and Zhang 2016). The combination of RS and field measurements contributes to a more robust, practical, and cost-effective approach for AGB estimations at

\footnotetext{
* Corresponding author. MED - Mediterranean Institute for Agriculture, Environment and Development, Departamento de Engenharia Rural, Escola Ciências e Tecnologia, Universidade de Évora, Pólo da Mitra, Ap. 94, 7006, Évora, Portugal.

E-mail addresses: pmrlourencov2@gmail.com (P. Lourenço), sgodinho@uevora.pt (S. Godinho).
} 